\title{
Comparison of Smallest Eigenvalues for Nabla Fractional Boundary Value Problems
}

\author{
Jagan Mohan Jonnalagadda \\ Department of Mathematics, \\ Birla Institute of Technology and Science Pilani, \\ Hyderabad - 500078, Telangana, India. \\ email: j.jaganmohan@hotmail.com
}

\begin{abstract}
In this article, we establish the existence of and then compare smallest eigenvalues for nabla fractional boundary value problems involving a fractional difference boundary condition, using the theory of $u_{0}$-positive operators with respect to a cone in a Banach space.
\end{abstract}

Key Words: Nabla fractional difference, boundary value problem, cone, $u_{0^{-}}$ positive operator, eigenvalue

AMS Classification: 26A33, 34B09, 39A12. 


\title{
Comparison of Smallest Eigenvalues for Nabla Fractional Boundary Value Problems
}

\author{
Jagan Mohan Jonnalagadda ${ }^{1}$
}

\begin{abstract}
In this article, we establish the existence of and then compare smallest eigenvalues for nabla fractional boundary value problems involving a fractional difference boundary condition, using the theory of $u_{0}$-positive operators with respect to a cone in a Banach space.
\end{abstract}

Key Words: Nabla fractional difference, boundary value problem, cone, $u_{0^{-}}$ positive operator, eigenvalue

AMS Classification: 26A33, 34B09, 39A12

\section{INTRODUCTION}

The theories of Krein-Rutman [30] and Krasnosel'skii [29] have been used by many authors to establish the existence of and then compare smallest eigenvalues of boundary value problems for differential equations [5, 10, 11, 12, 13, 32], difference equations $[7,16]$, dynamic equations on time scales $[6,19]$, fractional differential equations $[8,9,18,28]$, and delta fractional difference equations [17, 33, 34].

Motivated by these works, in this paper, we obtain the existence of and then compare smallest eigenvalues for the eigenvalue problems

$$
\begin{array}{ll}
\left(\nabla_{\rho(a)}^{\alpha} u\right)(t)+\lambda_{1} p(t) u(t)=0, & t \in \mathbb{N}_{a+2}^{b}, \\
\left(\nabla_{\rho(a)}^{\alpha} u\right)(t)+\lambda_{2} q(t) u(t)=0, & t \in \mathbb{N}_{a+2}^{b},
\end{array}
$$

satisfying the boundary conditions

$$
u(a)=\left(\nabla_{\rho(a)}^{\beta} u\right)(b)=0 .
$$

Here $1<\alpha<2,0 \leq \beta \leq 1, a, b \in \mathbb{R}$ with $b-a \in \mathbb{N}_{2}, p, q: \mathbb{N}_{a+2}^{b} \rightarrow$ $(0, \infty), \nabla_{\rho(a)}^{\alpha}$ and $\nabla_{\rho(a)}^{\beta}$ are $\alpha^{\text {th }}$ and $\beta^{\text {th }}$-order nabla fractional difference operators, respectively. Observe that the pair of boundary conditions in (1.3) reduces to conjugate $[3,14,24]$, right-focal [22] and right-focal type [23] boundary conditions as $\beta \rightarrow 0^{+}, \beta \rightarrow 1^{-}$and $\beta \rightarrow(\alpha-1)$, respectively.

This article is organized as follows: In Section 2, we state the preliminary definitions and results from nabla fractional calculus and the theory of $u_{0}$-positive operators with respect to a cone in a Banach space. In Section 3, we define the appropriate Banach space and establish the existence of and then compare smallest eigenvalues of (1.1) - (1.3) and (1.2) - (1.3).

\footnotetext{
${ }^{1}$ Department of Mathematics, Birla Institute of Technology and Science Pilani, Hyderabad 500078, Telangana, India. email: j.jaganmohan@hotmail.com
} 


\section{Preliminaries}

Denote the set of all real numbers by $\mathbb{R}$. For any $a, b \in \mathbb{R}$ such that $b-a \in \mathbb{N}_{1}$, define

$$
\mathbb{N}_{a}:=\{a, a+1, a+2, \ldots\} \text { and } \mathbb{N}_{a}^{b}:=\{a, a+1, a+2, \ldots, b\} .
$$

Assume that empty sums and products are taken to be 0 and 1 , respectively.

Definition 2.1 (See [4]). The backward jump operator $\rho: \mathbb{N}_{a} \rightarrow \mathbb{N}_{a}$ is defined by

$$
\rho(t)=\max \{a,(t-1)\}, \quad t \in \mathbb{N}_{a} .
$$

Definition 2.2 (See $[27,31]$ ). The Euler gamma function is defined by

$$
\Gamma(z):=\int_{0}^{\infty} t^{z-1} e^{-t} d t, \quad \Re(z)>0 .
$$

Using the reduction formula

$$
\Gamma(z+1)=z \Gamma(z), \quad \Re(z)>0,
$$

the Euler gamma function can be extended to the half-plane $\Re(z) \leq 0$ except for $z \neq 0,-1,-2, \ldots$

Definition 2.3 (See [15]). For $t \in \mathbb{R} \backslash\{\ldots,-2,-1,0\}$ and $r \in \mathbb{R}$ such that $(t+r) \in \mathbb{R} \backslash\{\ldots,-2,-1,0\}$, the generalized rising function is defined by

$$
t^{\bar{r}}=\frac{\Gamma(t+r)}{\Gamma(t)} .
$$

We use the convention that if $t \in\{\ldots,-2,-1,0\}$ and $r \in \mathbb{R}$ such that $(t+r) \in$ $\mathbb{R} \backslash\{\ldots,-2,-1,0\}$, then

$$
t^{\bar{r}}:=0 \text {. }
$$

Definition 2.4 (See [4]). Let $u: \mathbb{N}_{a} \rightarrow \mathbb{R}$ and $N \in \mathbb{N}_{1}$. The first order backward (nabla) difference of $u$ is defined by

$$
(\nabla u)(t):=u(t)-u(t-1), \quad t \in \mathbb{N}_{a+1},
$$

and the $N^{\text {th }}$-order nabla difference of $u$ is defined recursively by

$$
\left(\nabla^{N} u\right)(t):=\left(\nabla\left(\nabla^{N-1} u\right)\right)(t), \quad t \in \mathbb{N}_{a+N} .
$$

Definition 2.5 (See [15]). Let $u: \mathbb{N}_{a+1} \rightarrow \mathbb{R}$ and $N \in \mathbb{N}_{1}$. The $N^{\text {th }}$-order nabla sum of $u$ based at $a$ is given by

$$
\left(\nabla_{a}^{-N} u\right)(t):=\frac{1}{(N-1) !} \sum_{s=a+1}^{t}(t-\rho(s))^{\overline{N-1}} u(s), \quad t \in \mathbb{N}_{a},
$$

where by convention $\left(\nabla_{a}^{-N} u\right)(a)=0$. We define $\left(\nabla_{a}^{-0} u\right)(t):=u(t)$.

Definition 2.6 (See [15]). Let $u: \mathbb{N}_{a+1} \rightarrow \mathbb{R}$ and $\nu>0$. The $\nu^{\text {th }}$-order nabla sum of $u$ based at $a$ is given by

$$
\left(\nabla_{a}^{-\nu} u\right)(t):=\frac{1}{\Gamma(\nu)} \sum_{s=a+1}^{t}(t-\rho(s))^{\overline{\nu-1}} u(s), \quad t \in \mathbb{N}_{a}
$$


where by convention $\left(\nabla_{a}^{-\nu} u\right)(a)=0$.

Definition 2.7 (See [15]). Let $u: \mathbb{N}_{a+1} \rightarrow \mathbb{R}, \nu>0$ and choose $N \in \mathbb{N}_{1}$ such that $N-1<\nu \leq N$. Then, we define the $\nu^{\text {th }}$-order nabla fractional difference by

$$
\left(\nabla_{a}^{\nu} u\right)(t):=\left(\nabla^{N}\left(\nabla_{a}^{-(N-\nu)} u\right)\right)(t), \quad t \in \mathbb{N}_{a+N} .
$$

Definition 2.8 (See [29]). Let $\mathcal{B}$ be a real Banach space. A set $\mathcal{P} \subset \mathcal{B}$ is called a cone if the following conditions are satisfied:

(1) $\mathcal{P}$ is closed;

(2) if $u, v \in \mathcal{P}$ then $\alpha u+\beta v \in \mathcal{P}$ for all $\alpha, \beta \geq 0$;

(3) if $u \in \mathcal{P}$ and $-u \in \mathcal{P}$ then $u=0$.

A cone $\mathcal{P}$ is solid if the interior, $\mathcal{P}^{o}$, of $\mathcal{P}$, is nonempty. A cone is called reproducing if every element $w \in \mathcal{B}$ can be represented in the form

$$
w=u-v, \quad u, v \in \mathcal{P} .
$$

Remark 1 (See [29]). Every solid cone is reproducing.

By means of a cone, we define a partial ordering relation in a Banach space as follows.

Definition 2.9 (See [29]). Let $\mathcal{P}$ be a cone in a real Banach space $\mathcal{B}$. For all $u$, $v \in \mathcal{B}$, we write $u \leq v$ with respect to $\mathcal{P}$ if $v-u \in \mathcal{P}$.

Further, we also introduce a partial ordering relation on bounded linear operators defined on a Banach space.

Definition 2.10 (See [29]). Let $\mathcal{P}$ be a cone in a real Banach space $\mathcal{B}$ and $T$, $S: \mathcal{B} \rightarrow \mathcal{B}$ are bounded linear operators. We write $T \leq S$ with respect to $\mathcal{P}$ if $T u \leq S u$ for all $u \in \mathcal{P}$.

Definition 2.11 (See [29]). A bounded linear operator $T: \mathcal{B} \rightarrow \mathcal{B}$ is $u_{0}$-positive with respect to $\mathcal{P}$ if there exists $u_{0} \in \mathcal{P} \backslash\{0\}$ such that for each $u \in \mathcal{P} \backslash\{0\}$, there exist positive constants $k_{1}(u)$ and $k_{2}(u)$ such that $k_{1}\left(u_{0}\right) \leq T u \leq k_{2}\left(u_{0}\right)$ with respect to $\mathcal{P}$.

We use the following three theorems to establish our main results.

Theorem 2.1 (See [29]). Let $\mathcal{P} \subset \mathcal{B}$ be a solid cone. If $T: \mathcal{B} \rightarrow \mathcal{B}$ is a linear operator such that $T: \mathcal{P} \backslash\{0\} \rightarrow \mathcal{P}^{o}$, then $T$ is $u_{0}$-positive.

Theorem 2.2 (See [29]). Let $\mathcal{B}$ be a real Banach space, $\mathcal{P} \subset \mathcal{B}$ be a reproducing cone and $T: \mathcal{B} \rightarrow \mathcal{B}$ be a compact, $u_{0}$-positive linear operator. Then, $T$ has an essentially unique eigenvector in $\mathcal{P}$, and the corresponding eigenvalue is simple, positive, and larger than the absolute value of any other eigenvalue.

Theorem 2.3 (See [25]). Let $\mathcal{B}$ be a real Banach space, $\mathcal{P} \subset \mathcal{B}$ be a cone and $T$, $S: \mathcal{B} \rightarrow \mathcal{B}$ be bounded, linear operators. Assume at least one of the operators $T$ and $S$ is $u_{0}$-positive. If $T \leq S, T u_{1} \geq \lambda_{1} u_{1}$ for some $u_{1} \in \mathcal{P}$ and $\lambda_{1}>0$, and $S u_{2} \leq \lambda_{2} u_{2}$ for some $u_{2} \in \mathcal{P}$ and $\lambda_{2}>0$, then $\lambda_{1} \leq \lambda_{2}$. Furthermore, $\lambda_{1}=\lambda_{2}$ implies $u_{1}$ is a scalar multiple of $u_{2}$. 


\section{Main Results}

The author [21] has derived the Green's function $G(\beta ; t, s)$ for

$$
\left(\nabla_{\rho(a)}^{\alpha} u\right)(t)=0, \quad t \in \mathbb{N}_{a+2}^{b},
$$

satisfying (1.3) and also obtained a few of its properties.

$$
G(\beta ; t, s)= \begin{cases}\frac{1}{\Gamma(\alpha)}\left[\frac{(b-s+1)^{\overline{\alpha-\beta-1}}}{(b-a)^{\overline{\alpha-\beta-1}}}(t-a)^{\overline{\alpha-1}}\right], & t \in \mathbb{N}_{a}^{\rho(s)}, \\ \frac{1}{\Gamma(\alpha)}\left[\frac{(b-s+1)^{\overline{\alpha-\beta-1}}}{(b-a)^{\overline{\alpha-\beta-1}}}(t-a)^{\overline{\alpha-1}}-(t-s+1)^{\overline{\alpha-1}}\right], & t \in \mathbb{N}_{s}^{b} .\end{cases}
$$

Theorem 3.1 (See [21]). The Green's function $G(t, s)$ defined in (3.2) satisfies the following properties:

(1) $G(\beta ; a, s)=0$ for all $0 \leq \beta \leq 1$ and $s \in \mathbb{N}_{a+1}^{b}$.

(2) $G(0 ; b, s)=0$ for all $s \in \mathbb{N}_{a+1}^{b}$.

(3) $G(\beta ; t, a+1)=0$ for all $0 \leq \beta \leq 1$ and $t \in \mathbb{N}_{a}^{b}$.

(4) $G(0 ; t, s)>0$ for all $(t, s) \in \mathbb{N}_{a+1}^{b-1} \times \mathbb{N}_{a+2}^{b}$.

(5) $G(\beta ; t, s)>0$ for all $0<\beta \leq 1$ and $(t, s) \in \mathbb{N}_{a+1}^{b} \times \mathbb{N}_{a+2}^{b}$.

Observe that $u$ is a solution of (1.1) - (1.3) if and only if $u$ is a solution of the summation equation

$$
u(t)=\lambda_{1} \sum_{s=a+2}^{b} G(\beta ; t, s) p(s) u(s), \quad t \in \mathbb{N}_{a}^{b} .
$$

Similarly, $u$ is a solution of (1.2) - (1.3) if and only if $u$ is a solution of the summation equation

$$
u(t)=\lambda_{2} \sum_{s=a+2}^{b} G(\beta ; t, s) q(s) u(s), \quad t \in \mathbb{N}_{a}^{b}
$$

Denote by

$$
\mathcal{B}=\left\{u: \mathbb{N}_{a}^{b} \rightarrow \mathbb{R} \mid u(a)=\left(\nabla_{\rho(a)}^{\beta} u\right)(b)=0\right\} \subseteq \mathbb{R}^{b-a+1} .
$$

Clearly, $\mathcal{B}$ is a Banach space equipped with the maximum norm

$$
\|u\|:=\max _{t \in \mathbb{N}_{a}^{b}}|u(t)| .
$$

Define the cone

$$
\mathcal{P}=\left\{u \in \mathcal{B} \mid u(t) \geq 0 \text { for all } t \in \mathbb{N}_{a}^{b}\right\}
$$

Since

$$
\Omega=\left\{u \in \mathcal{B} \mid u(t)>0 \text { for all } t \in \mathbb{N}_{a+1}^{b-1}\right\} \subset \mathcal{P}^{o},
$$

$\mathcal{P}$ is solid and hence it is reproducing. Define the operators

$$
\begin{aligned}
& (T u)(t)=\sum_{s=a+2}^{b} G(\beta ; t, s) p(s) u(s), \quad t \in \mathbb{N}_{a}^{b}, \\
& (S u)(t)=\sum_{s=a+2}^{b} G(\beta ; t, s) q(s) u(s), \quad t \in \mathbb{N}_{a}^{b} .
\end{aligned}
$$


Clearly, $T, S: \mathcal{B} \rightarrow \mathcal{B}$ are linear. Note that $T$ and $S$ are summation operators on a discrete finite set. Hence, $T$ and $S$ are compact.

Lemma 3.2. The operators $T$ and $S$ are $u_{0}$-positive with respect to $\mathcal{P}$.

Proof. We prove this statement for the operator $T$. For this purpose, we apply Theorem 2.1. Clearly, $\mathcal{P} \subset \mathcal{B}$ is a solid cone and $T: \mathcal{B} \rightarrow \mathcal{B}$ is a linear operator. It is enough to show that $T: \mathcal{P} \backslash\{0\} \rightarrow \mathcal{P}^{o}$. To see this, let $u \in \mathcal{P} \backslash\{0\}$. Then, there exists a $t_{0} \in \mathbb{N}_{a+2}^{b-1}$ such that $u\left(t_{0}\right)>0$. Since $G(\beta ; t, s)>0$ for all $0 \leq \beta \leq 1$ and $(t, s) \in \mathbb{N}_{a+1}^{b-1} \times \mathbb{N}_{a+2}^{b}$ and $p(s)>0$ for all $s \in \mathbb{N}_{a+2}^{b}$, we have

$$
\begin{aligned}
(T u)(t) & =\sum_{s=a+2}^{b} G(\beta ; t, s) p(s) u(s) \\
& \geq G\left(\beta ; t, t_{0}\right) p\left(t_{0}\right) u\left(t_{0}\right)>0,
\end{aligned}
$$

for all $t \in \mathbb{N}_{a+1}^{b-1}$. So, $T u \in \Omega \subset \mathcal{P}^{o}$. The proof is complete.

Remark 2. Let $\lambda_{1}$ be a nonzero eigenvalue of (1.1) - (1.3). If $u$ is an eigenvector corresponding to $\lambda_{1}$ of (1.1) - (1.3), then

$$
\frac{1}{\lambda_{1}} u=T u
$$

So, the eigenvalues of (1.1) - (1.3) are reciprocals of the eigenvalues of (3.5), and conversely.

Theorem 3.3. $T$ has an essentially unique eigenvector $u \in \mathcal{P} \backslash\{0\}$, and the corresponding eigenvalue $\Lambda$ is positive, simple, and larger than the absolute value of any other eigenvalue.

Proof. We know that $\mathcal{P}$ is a reproducing cone and $T$ is a compact, $u_{0}$-positive linear operator. Then, by Theorem $2.2, T$ has an essentially unique eigenvector $u \in \mathcal{P} \backslash\{0\}$, and the corresponding eigenvalue $\Lambda$ is positive, simple, and larger than the absolute value of any other eigenvalue.

Remark 3. From the proof of Lemma 3.2, we observe that $(T u)(t)>0$ for all $t \in \mathbb{N}_{a+1}^{b-1}$ and hence $T u \in \mathcal{P}^{o}$. It follows from Theorem 3.3 that $\Lambda u=T u$. Thus, we obtain

for all $t \in \mathbb{N}_{a+1}^{b-1}$. Therefore, $u \in \Omega \subset \mathcal{P}^{o}$.

$$
u(t)=\frac{1}{\Lambda}(T u)(t)>0
$$

Theorem 3.4. Let $p(s) \leq q(s)$ for all $s \in \mathbb{N}_{a+2}^{b}$. Let $\Lambda_{1}$ and $\Lambda_{2}$ be the eigenvalues defined in Theorem 3.3 associated with $T$ and $S$, respectively, with the essentially unique eigenvectors $u_{1}$ and $u_{2}$ in $\mathcal{P} \backslash\{0\}$. Then, $\Lambda_{1} \leq \Lambda_{2}$. Furthermore, $\Lambda_{1}=\Lambda_{2}$ if and only if $p(s)=q(s)$ for all $s \in \mathbb{N}_{a+2}^{b}$.

Proof. Let $p(s) \leq q(s)$ for all $s \in \mathbb{N}_{a+2}^{b}$. Then, for any $u \in \mathcal{P}$ and $t \in \mathbb{N}_{a}^{b}$,

$$
(S u-T u)(t)=\sum_{s=a+2}^{b} G(\beta ; t, s)(q(s)-p(s)) u(s) \geq 0 \text {. }
$$


So, $(S u-T u) \in \mathcal{P}$ for all $u \in \mathcal{P}$. That is, $T \leq S$ with respect to $\mathcal{P}$. Then, by Theorem 2.3, we obtain $\Lambda_{1} \leq \Lambda_{2}$.

Now, we prove the second statement of the theorem. If possible, suppose $p\left(t_{0}\right)<$ $q\left(t_{0}\right)$, for some $t_{0} \in \mathbb{N}_{a+2}^{b-1}$. Since $u_{1} \in \mathcal{P}^{o}$, we have $u_{1}\left(t_{0}\right)>0$. Then, for all $t \in \mathbb{N}_{a+1}^{b-1}$,

$$
\begin{aligned}
\left(S u_{1}-T u_{1}\right)(t) & =\sum_{s=a+2}^{b} G(\beta ; t, s)(q(s)-p(s)) u_{1}(s) \\
& \geq G\left(\beta ; t, t_{0}\right)\left(q\left(t_{0}\right)-p\left(t_{0}\right)\right) u_{1}\left(t_{0}\right)>0
\end{aligned}
$$

implying that $\left(S u_{1}-T u_{1}\right) \in \Omega \subset \mathcal{P}^{o}$. So, there exists $\varepsilon>0$ such that $(S-T) u_{1}-$ $\varepsilon u_{1} \in \mathcal{P}$. Hence,

$$
\Lambda_{1} u_{1}+\varepsilon u_{1}=T u_{1}+\varepsilon u_{1} \leq S u_{1}
$$

which implies

$$
\left(\Lambda_{1}+\varepsilon\right) u_{1} \leq S u_{1}
$$

Since $S \leq S$ and $S u_{2}=\Lambda_{2} u_{2}$, by Theorem 2.3, we obtain

$$
\Lambda_{1}+\varepsilon \leq \Lambda_{2} \text { and } \Lambda_{1}<\Lambda_{2} \text {. }
$$

Thus, by contrapositive, if $\Lambda_{1}=\Lambda_{2}$, then $p(s)=q(s)$ for all $t \in \mathbb{N}_{a+2}^{b}$.

By Remark 2, the following theorem is an immediate consequence of Theorems 3.3 and 3.4 .

Theorem 3.5. Assume the hypotheses of Theorem 3.4. Then, there exist smallest positive eigenvalues $\lambda_{1}$ and $\lambda_{2}$ of (1.1) - (1.3) and (1.2) - (1.3), respectively, each of which is simple, positive, and less than the absolute value of any other eigenvalue of the corresponding problems. Also, eigenvectors corresponding to $\lambda_{1}$ and $\lambda_{2}$ may be chosen to belong to $\mathcal{P} \backslash\{0\}$. Then, $\lambda_{1} \geq \lambda_{2}$. Furthermore, $\lambda_{1}=\lambda_{2}$ if and only if $p(s)=q(s)$ for all $s \in \mathbb{N}_{a+2}^{b}$.

\section{REFERENCES}

[1] Abdeljawad, Thabet; Atıcı, Ferhan M. On the definitions of nabla fractional operators. Abstr. Appl. Anal. 2012, Art. ID 406757, 13 pp.

[2] Atıcı, Ferhan M.; Eloe, Paul W. Discrete fractional calculus with the nabla operator. Electron. J. Qual. Theory Differ. Equ. 2009, Special Edition I, No. 3, 12 pp.

[3] Brackins, Abigail; Boundary value problems of nabla fractional difference equations. Thesis (Ph.D.)-The University of Nebraska - Lincoln. 2014. 92 pp.

[4] Bohner, Martin; Peterson, Allan Dynamic equations on time scales. An introduction with applications. Birkhäuser Boston, Inc., Boston, MA, 2001. x+358 pp.

[5] Cheng, Sui Sun Comparison of eigenvalues for systems of linear differential equations. Proc. Amer. Math. Soc. 64 (1977), no. 1, 81-86.

[6] Chyan, Chuan Jen; Davis, John M.; Henderson, Johnny; Yin, William K. C. Eigenvalue comparisons for differential equations on a measure chain. Electron. J. Differential Equations 1998, No. 35, 7 pp.

[7] Davis, John M.; Eloe, Paul W.; Henderson, Johnny Comparison of eigenvalues for discrete Lidstone boundary value problems. Dynam. Systems Appl. 8 (1999), no. 3-4, 381-388. 
[8] Eloe, Paul W.; Neugebauer, Jeffrey T. Existence and comparison of smallest eigenvalues for a fractional boundary-value problem. Electron. J. Differential Equations 2014, No. 43, 10 pp.

[9] Eloe, Paul; Neugebauer, Jeffrey T. Smallest eigenvalues for a right focal boundary value problem. Fract. Calc. Appl. Anal. 19 (2016), no. 1, 11-18.

[10] Eloe, Paul W.; Henderson, Johnny Comparison of eigenvalues for a class of two-point boundary value problems. Appl. Anal. 34 (1989), 25-34.

[11] Eloe, Paul W.; Henderson, Johnny Comparison of eigenvalues for a class of multipoint boundary value problems. Recent trends in differential equations, 179-188, World Sci. Ser. Appl. Anal., 1, World Sci. Publ., River Edge, NJ, 1992.

[12] Gaudenzi, Marcellino Existence and comparison of eigenvalues for linear differential equations of order $n$. Dynam. Systems Appl. 7 (1998), no. 2, 187-214.

[13] Gentry, R. D.; Travis, C. C. Comparison of eigenvalues associated with linear differential equations of arbitrary order. Trans. Amer. Math. Soc. 223 (1976), 167-179.

[14] Gholami, Yousef; Ghanbari, Kazem Coupled systems of fractional $\nabla$-difference boundary value problems. Differ. Equ. Appl. 8 (2016), no. 4, 459-470.

[15] Goodrich, Christopher; Peterson, Allan C. Discrete fractional calculus. Springer, Cham, 2015.

[16] Hankerson, Darrel; Peterson, Allan Comparison of eigenvalues for focal point problems for $n$th order difference equations. Differential Integral Equations 3 (1990), no. 2, 363-380.

[17] Henderson, Johnny; Neugebauer, Jeffrey T. Smallest eigenvalues for a fractional difference equation with right focal boundary conditions. J. Difference Equ. Appl. 23 (2017), no. 8, $1317-1323$.

[18] Henderson, Johnny; Kosmatov, Nickolai Eigenvalue comparison for fractional boundary value problems with the Caputo derivative. Fract. Calc. Appl. Anal. 17 (2014), no. 3, 872880 .

[19] Hoffacker, J. Green's functions and eigenvalue comparisons for a focal problem on time scales. Advances in difference equations, IV. Comput. Math. Appl. 45 (2003), no. 6-9, 13391368 .

[20] A. Ikram, Green's functions and Lyapunov inequalities for nabla Caputo boundary value problems. Thesis (Ph.D.)-The University of Nebraska - Lincoln. 2018.

[21] Jonnalagadda, Jagan Mohan An ordering on Green's function and a Lyapunov-type inequality for a family of nabla fractional boundary value problems. Fract. Differ. Calc. 9 (2019), no. 1, 109-124.

[22] Jonnalagadda, Jagan Mohan Discrete fractional Lyapunov-type inequalities in nabla sense. Dyn. Contin. Discrete Impuls. Syst. Ser. A Math. Anal. Manuscript submitted for publication.

[23] Jonnalagadda, Jagan Mohan Lyapunov-type inequalities for discrete Riemann-Liouville fractional boundary value problems. Int. J. Difference Equ. 13 (2018), no. 2, 85-103.

[24] Jonnalagadda, Jagan Mohan On two-point Riemann-Liouville type nabla fractional boundary value problems. Adv. Dyn. Syst. Appl. 13 (2018), no. 2, 141-166.

[25] Keener, M. S.; Travis, C. C. Positive cones and focal points for a class of $n$ th-order differential equations. Trans. Amer. Math. Soc. 237 (1978), 331-351.

[26] Kelley, Walter G.; Peterson, Allan C. Difference equations. An introduction with applications. Second edition. Harcourt/Academic Press, San Diego, CA, 2001.

[27] Kilbas, Anatoly A.; Srivastava, Hari M.; Trujillo, Juan J. Theory and applications of fractional differential equations. North-Holland Mathematics Studies, 204. Elsevier Science B.V., Amsterdam, 2006.

[28] Koester, Angela M.; Neugebauer, Jeffrey T. Smallest eigenvalues for fractional boundary value problems with a fractional boundary condition. Journal Nonlinear Functional Analysis. 2017 (2017), Art. ID 1, 1-16. 
[29] Krasnosel'skiıi, M. A. Positive solutions of operator equations. Translated from the Russian by Richard E. Flaherty; edited by Leo F. Boron P. Noordhoff Ltd. Groningen 1964381 pp.

[30] Kreïn, M. G.; Rutman, M. A. Linear operators leaving invariant a cone in a Banach space. Amer. Math. Soc. Translation 1950, (1950). no. 26, 128 pp.

[31] Podlubny, Igor Fractional differential equations. An introduction to fractional derivatives, fractional differential equations, to methods of their solution and some of their applications. Mathematics in Science and Engineering, 198. Academic Press, Inc., San Diego, CA, 1999.

[32] Travis, Curtis C. Comparison of eigenvalues for linear differential equations of order $2 n$. Trans. Amer. Math. Soc. 177 (1973), 363-374.

[33] Yang, Aijun; Zhang, Li; Henderson, Johnny Comparison of smallest eigenvalues for fractional difference equations. Enlightenment Pure Appl. Math. 2 (2016), 161-170.

[34] Yang, Aijun; Zhang, Li; Henderson, Johnny Comparison of smallest eigenvalues for right focal Atici-Eloe fractional difference equations. J. Korean Soc. Math. Educ. Ser. B Pure Appl. Math. 24 (2017), no. 4, 191-200. 